\title{
Prediction of therapeutic effects of human cardiomyocytes in myocardial infarction using non-human primates model
}

\author{
Hyun-Min Cho, Je-Yoel Cho \\ Department of Biochemistry, BK21 PLUS Program for Creative Veterinary Science Research and Research Institute for Veterinary Science, College \\ of Veterinary Medicine, Seoul National University, Seoul, South Korea \\ Correspondence to: Je-Yoel Cho, DVM, PhD. Department of Biochemistry, College of Veterinary Medicine, Seoul National University, 1 Gwanak-ro, \\ Gwanak-gu, Seoul 08826, South Korea. Email: jeycho@snu.ac.kr. \\ Provenance: This is an invited Editorial commissioned by Section Editor Liuhua Zhou, MD (Department of Urology, Nanjing First Hospital, \\ Nanjing Medical University, Nanjing, China). \\ Comment on: Liu YW, Chen B, Yang X, et al. Human embryonic stem cell-derived cardiomyocytes restore function in infarcted hearts of \\ non-human primates. Nat Biotechnol 2018;36:597-605.
}

Submitted Sep 29, 2018. Accepted for publication Oct 11, 2018.

doi: 10.21037/atm.2018.10.33

View this article at: http://dx.doi.org/10.21037/atm.2018.10.33

Cardiovascular disease (CVD) is a critical disease that accounts for $34 \%$ of all deaths and consumes almost $\$ 300$ billion annually in the United States (1). Since myocardial infarction (MI) is the most severe and serious CVD with high lethality (2), attempts to overcome the disease are highly demanded. Although the development of pharmacological and intervention therapy enhances the survival rates, there are clear limitations to preventing or reversing the heart failure due to the massive muscle loss after MI. Currently, heart transplantation is considered to be the most fundamental treatment, but it also has many issues to resolve such as, complex post-operative management, very expensive strategies, and limited number of organ donors. Indeed, development of additional cardiovascular therapy is essential (3).

In spite of numerous therapeutic approaches that have been attempted using stem cells to treat CVD, few positive results and obvious limitations are reported in heart regeneration. Thus, there are crucial checkpoints to be confirmed before treating CVD through cell therapy. First, the transplanted cells must be differentiated into cardiac muscles. Second, the myotube formation with the cell graft and implanted heart, and eventually the electromechanical maturation of the graft with host myocardium must be confirmed, resulting in the transplantation of external cardiomyocytes which could restore the infarcted heart (3). When these prerequisites are confirmed, human pluripotent stem cells (hPSCs)-derived cardiac cells are ready to be engrafted into the injured heart and are expected to have a spontaneous action potential from the cell therapy (4).

In this study, Liu et al. demonstrated that transplantation of millions of cryopreserved human embryonic stem cellderived cardiomyocytes (hESC-CMs) successfully enhances cardiac function in macaque monkeys with large MI. The primate animal is the most suitable model for predictions in a clinical study due to being evolutionary adjacent and physiological similar to humans (5). After the hESCCMs injection in the macaque heart disease model, the authors confirmed the functional recovery and described it in two steps: (I) mechanical maturation without teratoma formation, and (II) lower burden of ventricular arrhythmia.

The authors induced larger infarcts in macaque hearts and showed the restoration effect of the transplanted hESC-CMs by cardiac magnetic resonance imaging (MRI) exclusively developed for macaque monkeys, which can provide the information related to left ventricular ejection fraction (LVEF), LV mass, the myocardial motion, as well as general information, such as heart structure (6). The results showed that hESC-CM transplantation improved $\sim 10 \%$ and $\sim 22 \%$ in LVEF and wall thickening, respectively. The enhancement of LVEF indicates an increased amount of blood released in each contraction and the enhanced efficiency of the heartbeat for systemic circulation (7). In addition, heart wall thickening could be interpreted as the 
loss of muscle due to the infarction being partially covered. However, to explain the therapeutic effect of transplanted cells in infarcted hearts, additional aspects of confirmation in histology are needed: (I) successful transplantation in infarcted region; (II) decreased scar formation; (III) sufficient maturation to function in macaque infarction model (8).

MI leads to massive loss of cardiomyocytes in the ischemic region and fibrotic scar formation, resulting in re-expression of heart failure (9). In regard to the success of transplantation aspect, the authors observed large grafts of myocardium (5.7$15.6 \%$ of the infarcted region) in the central infarcted region, using cardiac troponin 1 , and prevention of the wall thinning after 4 weeks of transplantation in the hESC-CM-treated group. Subsequently, the reduction of scar formation (from $25.2 \%$ to $19.9 \%$ of the LV in the hESC-CM-treated group compared to from $25.9 \%$ to $23.6 \%$ in the sham group) was histologically confirmed. Since the electromechanical maturation of the graft cells is very important to keep its beating regular and synchronized with the recipient's cardiac tissue (10), the authors confirmed the formation of mature $\mathrm{N}$-cadherin+ adherens junctions and Connexin 43+ gap junctions with the host heart. However, a minor limitation in the distribution of Connexin 43 remained. In addition, the authors could show vascularization with host cells by the graft using CD31+ in blood vessels, as well as proliferation of the graft cells using pericentriolar material-1 (PCM-1) and Ki67. Altogether, this data provided strong evidence that the implantation of hESC-CM reduced the fibrotic scar, filled up the muscle loss caused by the fibrosis process and could recover structural stability of the left ventricular wall. Meaningfully, the injected cardiomyocytes successfully formed a considerable maturation with the macaque infarcted heart.

The authors then investigated a new perspective on the cause of arrhythmias. An arrhythmia is an abnormal condition in which the heart contracts irregularly, and its severity presumes the degree of myocardial dysfunction. It has been known as a main limitation of cell-based therapy in heart disease because it has occurred when the external cells were implanted in an infarcted heart and has more frequently occurred in large rather than small animals $(11,12)$. Although its mechanism is unclear, differences of electrophysiologic maturity, gap junction isotypes, and wave propagation between the host heart and the graft are thought to be potential causes of arrhythmia $(4,5)$. Almeida et al. raised the possibility that the newly formed pacemaker from transplanted cells could be the cause of arrhythmia formation (13). From this point of view, Liu et al. performed an electronic mapping and determined that an ectopic activation source generated in transplanted hESCCMs improved the pulse generated by ectopic pacemaker cells. As mentioned above, since the stability is considered as the first priority in stem cell-mediated therapy (14), the authors excluded the chance of teratoma formation derived from undifferentiated pluripotent cells. In order to do that, the authors used cardiomyocytes fully differentiated by well-known methods and ultimately confirmed $86-99 \%$ of differentiation based on flow cytometry for cardiac troponin $\mathrm{T}$ and then confirmed the occurrence of teratoma by performing full necropsy on all animals with an experienced pathologist. As a result, teratoma was not found in any of the five hESC-CMs treated animals, except one macaque that had an extremely small carcinoma, which originated not from the grafted human cardiomyocytes but from the host macaque. It is suggested that the treatment of hESC$\mathrm{CM}$ for infarction may not induce teratoma or cancer by using almost fully differentiated cardiomyocytes.

In conclusion, the authors optimized the protocol on the cell type and dose, and the application time for hESC$\mathrm{CMs}$ and injected the cells into the macaque infarction model in order to introduce stem cells for cell therapy in further preclinical experiments and human clinical trials. Additionally, this study confirmed the therapeutic effects of hESC-CMs transplantation through electromechanical maturation and safety by showing the result without teratoma formation of transplanted cells. However, there is still a lot of room for improvement of cell therapy in heart disease. If the cell therapy taken in this study is tested with additional comprehensive treatments used in diverse heart diseases, it might improve the therapeutic effect. Additionally, tissue engineering with growth factors could be an alternative multidiscipline method, which could give an appropriate microenvironment for endogenous regeneration and could prevent the risk of cell injection. Furthermore, additional future efforts for enhancing these therapeutic effects might be gene editing by inserting the exotic genes such as VEGF and HGF, which could enhance the angiogenesis in infarcted region, and Akt, which could enhance the cell survival of transplanted cells (15-17). For that purpose, the gene editing technologies that are being developed recently such as clustered regularly interspaced short palindromic repeats- (CRISPR-) associated 9 (Cas9)mediated systems can be applied. The combined therapy using cardiomyocytes introduced by therapeutic genes could help cells to be more proliferative, and differentiate into the 
cardiomyocytes and to construct a better microenvironment in the MI region. There are still unsolved obstacles in the use of stem cell therapy in MI, but this study provided a good possibility in clinical therapy with a well-applied protocol utilizing hESC-CMs.

\section{Acknowledgements}

None.

\section{Footnote}

Conflicts of Interest: The authors have no conflicts of interest to declare.

\section{References}

1. Writing Group M, Mozaffarian D, Benjamin EJ, et al. Executive Summary: Heart Disease and Stroke Statistics--2016 Update: A Report From the American Heart Association. Circulation 2016;133:447-54.

2. Lazar E, Benedek T, Korodi S, et al. Stem cell-derived exosomes - an emerging tool for myocardial regeneration. World J Stem Cells 2018;10:106-15.

3. Michler RE. The current status of stem cell therapy in ischemic heart disease. J Card Surg 2018;33:520-31.

4. Chong JJ, Yang X, Don CW, et al. Human embryonicstem-cell-derived cardiomyocytes regenerate non-human primate hearts. Nature 2014;510:273-7.

5. Clarkson TB, Prichard RW, Morgan TM, et al. Remodeling of coronary arteries in human and nonhuman primates. JAMA 1994;271:289-94.

6. Mather AN, Fairbairn TA, Ball SG, et al. Reperfusion haemorrhage as determined by cardiovascular MRI is a predictor of adverse left ventricular remodelling and markers of late arrhythmic risk. Heart 2011;97:453-9.

7. Vourvouri EC, Poldermans D, Bax JJ, et al. Evaluation

Cite this article as: Cho HM, Cho JY. Prediction of therapeutic effects of human cardiomyocytes in myocardial infarction using non-human primates model. Ann Transl Med 2018;6(Suppl 1):S64. doi: 10.21037/atm.2018.10.33 of left ventricular function and volumes in patients with ischaemic cardiomyopathy: gated single-photon emission computed tomography versus two-dimensional echocardiography. Eur J Nucl Med 2001;28:1610-5.

8. Oh H, Ito H, Sano S. Challenges to success in heart failure: Cardiac cell therapies in patients with heart diseases. J Cardiol 2016;68:361-7.

9. Tani H, Sadahiro T, Ieda M. Direct Cardiac Reprogramming: A Novel Approach for Heart Regeneration. Int J Mol Sci 2018;19.

10. Morgan KY, Black LD 3rd. Mimicking isovolumic contraction with combined electromechanical stimulation improves the development of engineered cardiac constructs. Tissue Eng Part A 2014;20:1654-67.

11. Lalit PA, Hei DJ, Raval AN, et al. Induced pluripotent stem cells for post-myocardial infarction repair: remarkable opportunities and challenges. Circ Res 2014;114:1328-45.

12. Macia E, Boyden PA. Stem cell therapy is proarrhythmic. Circulation 2009;119:1814-23.

13. Almeida SO, Skelton RJ, Adigopula S, et al. Arrhythmia in stem cell transplantation. Card Electrophysiol Clin 2015;7:357-70.

14. Masuda S, Miyagawa S, Fukushima S, et al. Emerging innovation towards safety in the clinical application of ESCs and iPSCs. Nat Rev Cardiol 2014;11:553-4.

15. Yu H, Lu K, Zhu J, et al. Stem cell therapy for ischemic heart diseases. Br Med Bull 2017;121:135-54.

16. Cho HM, Kim PH, Chang HK, et al. Targeted Genome Engineering to Control VEGF Expression in Human Umbilical Cord Blood-Derived Mesenchymal Stem Cells: Potential Implications for the Treatment of Myocardial Infarction. Stem Cells Transl Med 2017;6:1040-51.

17. Chang HK, Kim PH, Cho HM, et al. Inducible HGFsecreting Human Umbilical Cord Blood-derived MSCs Produced via TALEN-mediated Genome Editing Promoted Angiogenesis. Mol Ther 2016;24:1644-54. 